\title{
THE INTRINSIC EQUATION FOR EULER'S RESISTANCE INTEGRAL.
}

BY PROFESSOR G. H. LIGHT.

Euler, in his Scientia Navalis, Proposition 53, gives a problem whose solution leads to the resistance integral

$$
I=\int_{t_{0}}^{t_{1}} \frac{y^{\prime 3}}{x^{\prime 2}+y^{\prime 2}} d t
$$

This problem is as follows: Among all curves $A M$ which with the axis $A P$ and perpendicular $P M$ comprehend the same area; to find that one which with its symmetric branch on the opposite side of the axis $A P$ will form the figure offering the least resistance in water, when it moves in the direction $P A$ along the axis.

This is a problem of the isoperimetric type where the integral $I$ to be minimized is the integral (1) and the condition imposed is that the area between the axis and the curve is fixed, i. e.,

$$
G=\int_{t_{0}}^{t_{1}} y x^{\prime} d t=l .
$$

The solution of this problem is known to be

$$
x=\frac{1}{\lambda} \frac{p^{2}-1}{\left(p^{2}+1\right)^{2}}+\alpha, \quad y=\frac{1}{\lambda} \frac{2 p^{3}}{\left(p^{2}+1\right)^{2}}+\beta,
$$

where $p=y^{\prime} \mid x^{\prime}$ and $\alpha, \lambda$ and $\beta$ are constants. Since this curve can always be obtained by a similarity transformation from the curve

$$
x=\frac{p^{2}-1}{\left(p^{2}+1\right)^{2}}, \quad y=\frac{2 p^{3}}{\left(p^{2}+1\right)^{2}},
$$

the general properties of the extremal can be obtained from the latter.

So far as is known, the exact nature of the extremals has never been determined. It is simply stated that they are rational curves of the fourth order with three cusps. The object of this paper is to show that when their intrinsic 
equation is obtained, it is seen that the curve is a hypocycloid of three cusps. This is deduced in the following way:

The radius of curvature is found from (2) to be

$$
R=\frac{2 p\left(3-p^{2}\right)}{\left(1+p^{2}\right)^{3 / 2}}
$$

The length of arc is

$$
S=\int_{0}^{p} \frac{2 p\left(3-p^{2}\right)}{\left(1+p^{2}\right)^{5 / 2}} d p=-\frac{8}{3} \frac{1}{\left(1+p^{2}\right)^{3 / 2}}+\frac{2}{\left(1+p^{2}\right)^{1 / 2}}+\frac{2}{3},
$$

from which

$$
\frac{3}{2}\left(S-\frac{2}{3}\right)=\frac{3 p^{2}-1}{\left(1+p^{2}\right)^{3 / 2}} .
$$

In order to eliminate $p$ from equations (3) and (4), form the expressions $R^{2} / 4$ and $\frac{9}{4}\left(S-\frac{2}{3}\right)^{2}$. It is seen at once that

(5) $\frac{R^{2}}{4}+\frac{9}{4}\left(S-\frac{2}{3}\right)^{2}=1$, or $R^{2}+9\left(S-\frac{2}{3}\right)^{2}=4$,

which is the intrinsic equation of a hypocycloid. The curvature of (2) is

also

$$
k=\frac{\left(1+p^{2}\right)^{3 / 2}}{2 p\left(3-p^{2}\right)},
$$

$$
\frac{d x}{d p}=\frac{2 p\left(3-p^{2}\right)}{\left(1+p^{2}\right)^{3}}, \quad \frac{d y}{d p}=\frac{2 p^{2}\left(3-p^{2}\right)}{\left(1+p^{2}\right)^{3}} .
$$

From (6) and (7) it is seen that when $p=0$ or $\pm \sqrt{3}, d x / d p$ and $d y / d p$ become zero and $k=\infty$. Hence (5) is the equation of a hypocycloid of three cusps.

University of Colorado.

\section{HERMITE'S WORKS.}

Euvres de Charles Hermite. Publiées sous les auspices de l'Académie des Sciences par Emir.e Picard. Vol. IV. Paris, Gauthier-Villars, 1917. 8vo. 593 pp.

THE present volume brings to a close the CEuvres of Hermite. It contains about ninety papers arranged chronologically, dating from 1879 and continuing to the year of his death, 1901. 